\title{
External fixation-assisted reduction for the treatment of neglected hip dislocations with limb length discrepancy: a retrospective study of 13 cases
}

\author{
Pengyu $\mathrm{Li}^{\dagger}$, Fulin Tao ${ }^{\dagger}$, Wenhao Song, Jinlei Dong, Daodi Qiu*i] and Dongsheng Zhou*
}

\begin{abstract}
Background: The purpose of this study was to evaluate a new method for treating neglected hip dislocation with limb length discrepancy by using external fixation-assisted pre-reduction.

Methods: Thirteen patients admitted between January 2010 to February 2018 with a mean duration from injury to surgery of $5.0 \pm 2.1$ months and an average preoperative leg-length discrepancy of $7.7 \pm 2.3 \mathrm{~cm}$ were enrolled in this study. The dislocation and associated acetabular fracture type, clinical outcomes and residual limb length discrepancy were evaluated.

Results: All patients had posterior dislocations, and nine patients presented with acetabular fractures and were followed-up for at least 12 months. The average traction duration of external fixators was $28.8 \pm 8.0$ days and all patients received second-stage open reduction and internal fixation. Six patients showed residual limb length discrepancy within $2 \mathrm{~cm}$. Patients showed significant improvement in hip function and pain relief. Complications including avascular femoral head necrosis and osteoarthritis occurred in three patients.

Conclusion: Effective correction of limb length discrepancy and improved function were observed in patients with neglected hip dislocations and limb equality using traction by external fixation combined with second-stage open reduction. Further follow-up is required to determine long-term outcomes.
\end{abstract}

Keywords: Neglected hip dislocation, Limb length discrepancy, External fixation

\section{Background}

Traumatic hip dislocation is usually caused by highenergy trauma and accounts for $5 \%$ of all traumatic joint dislocations [1]. Most dislocations are caused by motor vehicle accidents and associated with unfastened safety belts [2]. Early recognition and a prompt reduction is of great importance $[3,4]$ because delayed diagnosis may lead to long-term complications including avascular necrosis of the femoral head (AVN) and osteoarthritis [5]. Neglected hip dislocations are rare in adults, especially in highincome countries [6]. However, in developing countries, patients may not seek medical attention or visit the

\footnotetext{
*Correspondence: qiudaodifly@163.com; sdgkxh@126.com

${ }^{\dagger}$ Pengyu Li and Fulin Tao contributed equally to this work.

Department of Orthopedic Surgery, Shandong Provincial Hospital Affiliated

to Shandong University, 324 Jingwu Road, Jinan, Shandong, China
}

hospital many days after trauma because of financial strain and neglected hip dislocations are not uncommon [7]. The current literature is limited on this subject.

Common clinical manifestations of neglected hip dislocation are shortening of the limb and limited function of the hip joint. For patients with neglected fracture, callus formation and adhesions caused by connective tissue make regular reduction difficult. The common treatments reported in previous studies for neglected hip dislocation included skeletal traction and open reduction. These treatments are associated with unsatisfactory outcomes and a high incidence of post-operative complications such as nerve injury, AVN, and osteoarthritis [8, 9]. Garrett et al. recommended total hip replacement (THR) for hip dislocations with a duration of $>3$ months [10], but others have reported that limited correction of limb length

(c) The Author(s). 2019 Open Access This article is distributed under the terms of the Creative Commons Attribution 4.0 International License (http://creativecommons.org/licenses/by/4.0/), which permits unrestricted use, distribution, and reproduction in any medium, provided you give appropriate credit to the original author(s) and the source, provide a link to the Creative Commons license, and indicate if changes were made. The Creative Commons Public Domain Dedication waiver (http://creativecommons.org/publicdomain/zero/1.0/) applies to the data made available in this article, unless otherwise stated. 
discrepancy may be achieved with THR [11, 12]. Considering that the best treatment for neglected hip dislocation remains controversial and to improve clinical outcome, we propose a new surgical strategy to treat neglected hip dislocations using external fixators for preoperative traction. The purpose of this study was to assess the utility of external fixation as a means for reducing the hip joint prior to second stage open reduction and internal fixation.

\section{Patients and methods}

We reviewed all patients with neglected hip dislocation treated in our department from January 2010 to February 2018 and identified twenty patients. Inclusion criteria were: (1) neglected hip dislocation of which duration from trauma to surgery was more than 3 weeks, (2) treated with external fixators, (3) $18<$ age $<60$. Seven patients were excluded because they were treated by skeletal traction or other surgery. In total, thirteen patients were enrolled in this study. Permission for this retrospective study was obtained from the Medical Ethics Committee of the authors' institution, and written informed consent was obtained from every patient. All patients suffered posterior dislocation and the classification has been described by Thompson and Epstein [13]. The associated acetabular fracture has been classified by Judet-Letournel [14]. The function of the hip joint was evaluated using the modified Merle D'Aubigné and
Postel scoring system [15], and clinical outcome was graded as follows: excellent (18), good (15-17), fair (13-14), and poor $(<14)$. Residual pain was assessed according to the visual analogue scale (VAS). Leg length was measured from the umbilicus to the medial malleolus by clinical examination. Anticoagulation was used from the time external fixation was performed until patient was able to begin mobilization or get out of bed. Patients began weight bearing 3 weeks after second-stage open reduction. Statistical testing was performed with the unpaired $\mathrm{t}$-test, and statistical difference was defined as $P<0.05$.

\section{Surgical procedures}

All surgeries were performed by a single experienced orthopedic surgeon. First-stage traction by external fixation: surgeries were performed on patients under regional anesthesia. Two incisions of approximately $1 \mathrm{~cm}$ were made on the iliac crest and lateral thigh. Two screws were placed in the anterior inferior iliac spine, and another two screws were placed in the femur middiaphysis under fluoroscopic guidance. A unilateral external fixator was then connected (Fig. 1). The traction procedure began 3 days after surgery, with the external fixator stretching $1-3 \mathrm{~mm}$ a day. A plain radiograph was taken every 5-7 days to examine the reduction. Traction was ceased when the femoral head was drawn beneath the articular surface of the acetabulum.

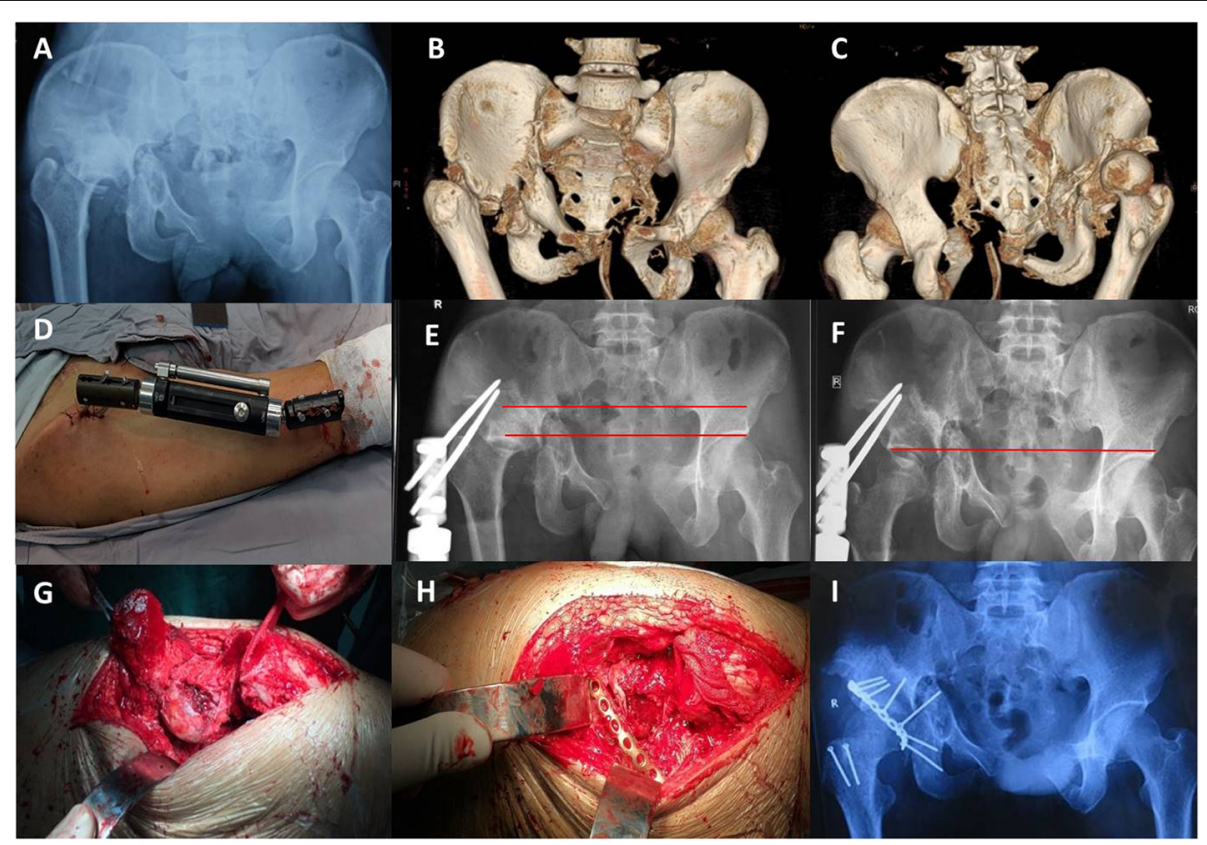

Fig. 1 Patient 12. a-c Preoperative X-ray and three-dimensional reconstruction computed tomography images 6 months after injury. $\mathbf{d}$-e External fixation placement. $\mathbf{f}$ The femoral head was drawn underneath the articular surface after 31 days of traction. $\mathbf{g}-\mathbf{h}, \mathbf{k}-\mathbf{I}$ incision, open reduction with osteotomy of the greater trochanter, and fixation of posterior wall of acetabulum. i Postoperative X-ray image showed correction of limb length discrepancy and reduction of femoral head was originated from our previous study [16] and was authorized by Chinese Journal of Orthopaedic Trauma 
Second-stage open reduction: after the first-stage traction, open reduction was performed under general anesthesia. A Kocher-Langenbeck (K-L) approach or combined ilioinguinal and K-L approaches were utilised. After superficial and deep dissection, a trochanteric flip osteotomy was performed when the acetabular roof fracture was present to allow for adequate exposure of the hip capsule. Capsulotomy was then performed, followed by clearing of fibrous tissue and intra-articular fragments, and finally a reduction of the femoral head. The malunion of posterior acetabular wall was then reconstructed and fixed with cannulated screws and a $3.5 \mathrm{~mm}$ reconstruction plate. An autogenous iliac bone graft was used when needed. The osteotomized trochanter was fixed with 2-3 small fragment cortex screws. Postoperative skeletal traction was used depending on the stability of fracture fixation after open reduction.

\section{Results}

Thirteen patients (five women, eight men) with an average age of 36.7 years (range, 19-49 years) were enrolled in this study. Nine patients had associated acetabular fractures, and the pattern of fracture and posterior dislocation are shown in Table 1. The mean duration from injury to surgery was 5.0 months (range, 2.5-9 months). The mean preoperative leg-length discrepancy was $7.7 \mathrm{~cm}$ (range, $5-12.2 \mathrm{~cm}$ ). Six patients presented with a postoperative leg-length discrepancy within $2 \mathrm{~cm}$ (Table 2). The average traction duration of external fixators was 28.8 days (range, 17-46 days). The mean operation time was $2.7 \pm$
$0.8 \mathrm{~h}$, and the average blood loss during the surgery was $1067.4 \pm 374.5 \mathrm{~mL}$. All patients were followed up for at least 12 months, with a mean follow-up duration of 15.4 months (range, 12-18 months). The mean Merle d'Aubigné score improved significantly from $5.1 \pm 1.7$ to $14.4 \pm$ 2.0 after surgery $(p<0.01)$. The mean preoperative VAS score was $4.3 \pm 1.3$, and the mean postoperative score was $0.9 \pm 1.0(\mathrm{p}<0.01)$, with five patients completely free of pain. No neurovascular injuries related to limb-lengthening occurred in these patients. Complications occurred in three patients during the follow-up, two patients had AVN, and one patient had osteoarthritis of the hip joint. None of the patients received further treatment at the last follow-up.

\section{Discussion}

Neglected hip dislocations often occur in children and are rarely reported in adults. This study showed effective correction of limb length discrepancy and significant functional improvement with external fixation-assisted reduction in thirteen adult patients. Though skeletal traction is important in joint reduction, the acetabulum becomes filled with fibrous tissue in neglected dislocations would make reduction difficult by simple traction. Skin traction usually carries a weight under $5 \mathrm{~kg}$. Halofemoral and tibial tubercle traction can carry more weight but it often ends with unsatisfactory results in patients with neglected hip dislocation $[9,17]$. Prolonged dislocation may cause severe joint contracture so it is difficult to achieve satisfactory reduction by regular

Table 1 Patients' detail

\begin{tabular}{|c|c|c|c|c|c|c|c|}
\hline Patient & $\begin{array}{l}\text { Duration from trauma } \\
\text { to surgery(months) }\end{array}$ & Acetabular fracture type & $\begin{array}{l}\text { Posterior dislocation } \\
\text { type }\end{array}$ & $\begin{array}{l}\text { Follow-up } \\
\text { (months) }\end{array}$ & $\begin{array}{l}\text { Clinical } \\
\text { outcome }\end{array}$ & VAS & Complication \\
\hline 1 & 5 & No & I & 15 & Good & 1 & \\
\hline 2 & 4.5 & No & I & 13 & Good & 0 & \\
\hline 3 & 9 & No & । & 18 & Fair & 1 & \\
\hline 4 & 5.5 & No & I & 14 & Good & 0 & \\
\hline 5 & 3 & Posterior wall & I & 15 & Excellent & 0 & \\
\hline 6 & 8 & Posterior wall & $\|$ & 17 & Poor & 2 & AVN \\
\hline 7 & 4 & Transverse with posterior wall & $\|$ & 17 & Fair & 3 & AVN \\
\hline 8 & 3 & posterior wall & $\|$ & 15 & Good & 0 & \\
\hline 9 & 2.5 & $\begin{array}{l}\text { Posterior column with posterior } \\
\text { wall }\end{array}$ & III & 18 & Poor & 2 & \\
\hline 10 & 2 & Posterior wall & III & 15 & Good & 0 & \\
\hline 11 & 7 & Posterior wall & III & 14 & Poor & 1 & $\mathrm{OA}$ \\
\hline 12 & 5 & Transverse with posterior wall & IV & 13 & Good & 1 & \\
\hline 13 & 6 & $\begin{array}{l}\text { Posterior column with posterior } \\
\text { wall }\end{array}$ & IV & 16 & Fair & 1 & \\
\hline
\end{tabular}

AVN Avascular femoral head necrosis 
Table 2 Correction of limb discrepancy

\begin{tabular}{|c|c|c|c|}
\hline \multirow[t]{2}{*}{ Patient } & \multicolumn{2}{|c|}{ Inequality of lower limb(cm) } & \multirow{2}{*}{$\begin{array}{l}\text { Traction } \\
\text { duration(days) }\end{array}$} \\
\hline & Preoperative & Postoperative & \\
\hline 1 & 5.6 & 0 & 17 \\
\hline 2 & 5.4 & 0 & 21 \\
\hline 3 & 8.2 & 1 & 27 \\
\hline 4 & 5 & 0 & 25 \\
\hline 5 & 7.5 & 0 & 25 \\
\hline 6 & 8.8 & 1.5 & 34 \\
\hline 7 & 12.2 & 2 & 46 \\
\hline 8 & 6.0 & 0 & 27 \\
\hline 9 & 9.4 & 2 & 35 \\
\hline 10 & 5.3 & 0 & 20 \\
\hline 11 & 11 & 1 & 38 \\
\hline 12 & 7 & 1.5 & 31 \\
\hline 13 & 8.2 & 0 & 28 \\
\hline
\end{tabular}

traction. Furthermore, heavy traction may lead to neurovascular complications.

Common complications of traumatic hip dislocation include infection, sciatic nerve palsy, AVN, heterotopic ossification, and post-traumatic arthritis [18]. Previous studies have reported that the incidence of arthritis was $16-30 \%$, and $8.1-10 \%$ for AVN in the mid-to-long-term follow-up $[1,19]$. The blood supply to femoral head is often damaged by traumatic dislocation, especially the medial femoral circumflex artery. Vascular compromise leads to intravascular coagulation and ischemic necrosis, resulting in chondral failure and accelerated joint degeneration [20]. A high incidence of osteonecrosis has been reported from 10 to $25 \%$ in hip dislocations [21]. Furthermore, the severity of the injury and time to reduction are associated with increased risks of osteonecrosis [20]. The rate of osteonecrosis is only $10 \%$ in adults after simple dislocation, but reaches $70 \%$ if the dislocation is accompanied by severe bony destruction such as fracture of the femoral head and acetabulum [22]. Hougaard and Thomsen reported that the rate of osteonecrosis of the femoral head can be reduced from 58 to $4.8 \%$ if hip reduction is performed within the first $6 \mathrm{~h}$ following injury [23]. Others have concluded a trend for decreased osteonecrosis of the femoral head when hip reduction was performed within $12 \mathrm{~h}$ [1]. In our patients, AVN occurred in 2 patients (15\%), and osteoarthritis occurred in 1 patient (7.7\%). All 3 patients suffered acetabular fractures with an average duration from injury to surgery of 6.3 months (range, 4 to 8 months). Patient 6 (Table 1) showed AVN 10 months after the surgery, and the Merle d'Aubigne score was improved from 4 to 12 . Patient 7 (Table 1) showed AVN 12 months after surgery, and the Merle d'Aubigne score was improved from 5 to 13.
Patient 11 (Table 1) showed osteoarthritis at the last follow-up (14 months), and the score was improved from 4 to 11 . Though complications occurred, patients demonstrated significant functional improvement. Previous studies have also reported that complications continue to occur within 5 years $[7,24]$, though our study only reports a short-term follow-up result within 18 months. As the incidence of secondary complications may increase with time [25], an accurate complication rate should be recalculated for long-term follow-up.

THR has been recommended for hip dislocations with a duration of more than 3 months [10], but usually provided limited correction of limb length discrepancy within $6 \mathrm{~cm}[11,12]$ and muscle release may be needed for improved correction [26]. It may provide limited efficacy for patients in this case series which all suffered limb inequality of more than $5 \mathrm{~cm}$. Considering all patients were under 50 years and no signs of necrosis or arthritis occurred when on admission, we decided to perform open reduction and fixation after traction. Follow-up results showed significant pain relief and function improvement. The satisfactory rate of clinical outcome was $53.8 \%$ (excellent and good results). Limb length discrepancy was effectively corrected, and no patient was left with $>2 \mathrm{~cm}$ of inequality, thereby laying the foundation for possible THR in the future. AVN and arthritis occurred in three patients. Though they showed unsatisfactory outcomes (two poor and one fair), none of them received further treatment at the last follow-up. A further study evaluating the long-term clinical outcomes of external fixation with delayed open reduction against total hip replacement is required.

From our experiences, external fixation was effective for pre-reduction in patients suffering from neglected hip dislocations with limb length discrepancy, but still several contraindications are noteworthy. First, this treatment option should not be used in patients with heterotopic ossification, which most commonly occurs in the hip joint, and the incidence after traumatic dislocation was 32 to $37 \%$ [27, 28]. Traction would be resisted by ectopic bone. As the fixation technique requires stable anchors, it is unfit for patients with an unstable pelvic ring or femur. Furthermore, patients with osteoporosis should not be considered because osteoporotic bone may fail under power of traction.

There are limitations in this study including the retrospective design and a small number of patients. This study did not evaluate long-term clinical outcomes or compare the treatment strategy with other techniques. Further studies are required for this purpose.

\section{Conclusions}

This retrospective review of 13 patients with neglected hip dislocation demonstrates the utility of a two-stage 
treatment regime, consisting of initial external fixation to reduce the hip joint followed by definitive open reduction and internal fixation of any associated fractures. This treatment regime resulted in improvements in leg length discrepancy, hip function and pain scores at a mean short-term follow-up of 15.4 months. Potential complications included avascular necrosis of the femoral head and post-traumatic osteoarthritis. Further studies are required to evaluate the treatment protocol against other options such as total hip replacement with longterm follow up.

\section{Abbreviations \\ AVN: Avascular necrosis of the femoral head; K-L: Kocher-Langenbeck; THR: Total hip replacement; VAS: Visual analogue scale}

\section{Acknowledgments}

No

\section{Authors' contributions}

DZ and DQ generated the conception of the study and designed the study. The acquisition, analysis and interpretation of data were done by $\mathrm{PL}, \mathrm{WS}$ and JD. PL and FT drafted the manuscript. DQ and DZ revised the final draft. All of authors have approved the version to be submitted and any substantially modified version that involves the author's contribution to the study. All of authors have agreed both to be personally accountable for the author's own contributions and to ensure that questions related to the accuracy or integrity of any part of the work are appropriately investigated and resolved.

\section{Funding}

This study was supported by the National Natural Science Foundation of China (No. 81301556). The funding body did not take part in the design of the study and collection, analysis, and interpretation of data, or in the writing of the manuscript.

\section{Availability of data and materials}

The data used in the current study are available from the corresponding author on reasonable request.

\section{Ethics approval and consent to participate}

The study was approved by The Medical Ethical Committee of Shandong Provincial Hospital Affiliated to Shandong University. The written informed consent was obtained from all patients included in this study.

\section{Consent for publication}

Not applicable.

\section{Competing interests}

The authors declare that they have no competing interests.

Received: 18 June 2019 Accepted: 19 December 2019

Published online: 26 December 2019

\section{References}

1. Sahin V, Karakas ES, Aksu S, Atlihan D, Turk CY, Halici M. Traumatic dislocation and fracture-dislocation of the hip: a long-term follow-up study. J Trauma. 2003;54(3):520-9.

2. Reigstad A. Traumatic dislocation of the hip. J Trauma. 1980;20(7):603-6.

3. Yang RS, Tsuang YH, Hang YS, Liu TK. Traumatic dislocation of the hip. Clin Orthop Relat Res. 1991;265:218-27.

4. Pietrafesa CA, Hoffman JR. Traumatic dislocation of the hip. Jama. 1983; 249(24):3342-6.

5. Zhou Y, Zhang C, Zhao S, Wang Q. Closed reduction of the traumatic posterior-dislocation of hip joint using a novel sitting technique: a case series. Medicine (Baltimore). 2018;97(41):e12538.

6. Young S, Banza L. Neglected traumatic anterior dislocation of the hip. Open reduction using the Bernese trochanter flip approach - a case report. Acta Orthop. 2017;88(3):348-50.
7. Hougaard K, Thomsen PB. Traumatic posterior fracture-dislocation of the hip with fracture of the femoral head or neck, or both. J Bone Joint Surg Am. 1988;70(2):233-9.

8. Banskota AK, Spiegel DA, Shrestha S, Shrestha OP, Rajbhandary T. Open reduction for neglected traumatic hip dislocation in children and adolescents. J Pediatr Orthop. 2007;27(2):187-91.

9. Gupta RC, Shravat BP. Reduction of neglected traumatic dislocation of the hip by heavy traction. J Bone Joint Surg Am. 1977;59(2):249-51.

10. Garrett JC, Epstein HC, Harris WH, Harvey JP Jr, Nickel VL. Treatment of unreduced traumatic posterior dislocations of the hip. J Bone Joint Surg Am. 1979:61(1):2-6.

11. Ilyas I, Rabbani SA. Total hip arthroplasty in chronic unreduced hip fracturedislocation. J Arthroplasty. 2009;24(6):903-8

12. Paavilainen T, Hoikka V, Solonen KA. Cementless total replacement for severely dysplastic or dislocated hips. J Bone Joint Surg. 1990;72(2):205-11.

13. Thompson VP, Epstein HC. Traumatic dislocation of the hip; a survey of two hundred and four cases covering a period of twenty-one years. J Bone Joint Surg Am. 1951;33-a(3):746-78 passim.

14. Judet R, Judet J, Letournel E. Fractures of the acetabulum: classification and surgical approaches for open reduction. Preliminary report. J Bone Joint Surg Am. 1964:46:1615-46.

15. Charnley J. The long-term results of low-friction arthroplasty of the hip performed as a primary intervention. J Bone Joint Surg. 1972;54(1):61-76.

16. Qiu D, Zhou D, et al. Application of 3D printing technique in treatment of obsolete pelvic and acetabular fractures. Chin J Orthop Trauma. 2017;19(7): $624-9$

17. Pai VS, Kumar B. Management of unreduced traumatic posterior dislocation of the hip: heavy traction and abduction method. Injury. 1990;21(4):225-7.

18. Nicholson JA, Scott CEH, Annan J, Ahmed I, Keating JF. Native hip dislocation at acetabular fracture predicts poor long-term outcome. Injury. 2018:49(10):1841-7.

19. Pascarella R, Fantasia R, Sangiovanni P, Maresca A, Massetti D, Politano R, et al. Traumatic hip fracture-dislocation: a middle-term follow up study and a proposal of new classification system of hip joint associated injury. Injury. 2019:50(4):S11-S20.

20. Begly JP, Robins B, Youm T. Arthroscopic treatment of traumatic hip dislocation. J Am Acad Orthop Surg. 2016:24(5):309-17.

21. Zalavras CG, Lieberman JR. Osteonecrosis of the femoral head: evaluation and treatment. J Am Acad Orthop Surg. 2014;22(7):455-64.

22. Duncan CP, Shim SS. Blood supply of the head of the femur in traumatic hip dislocation. Surg Gynecol Obstet. 1977;144(2):185-91.

23. Hougaard K, Thomsen PB. Traumatic posterior dislocation of the hip-prognostic factors influencing the incidence of avascular necrosis of the femoral head. Arch Orthop Trauma Surg. 1986:106(1):32-5.

24. Epstein HC, Wiss DA, Cozen L. Posterior fracture dislocation of the hip with fractures of the femoral head. Clin Orthop Relat Res. 1985:201:9-17.

25. Upadhyay SS, Moulton A, Srikrishnamurthy K. An analysis of the late effects of traumatic posterior dislocation of the hip without fractures. J Bone Joint Surg. 1983;65(2):150-2.

26. Harley JM, Wilkinson JA. Hip replacement for adults with unreduced congenital dislocation. A new surgical technique. J Bone Joint Surg. 1987; 69(5):752-5.

27. Ross JR, Schoenecker PL, Clohisy JC. Surgical dislocation of the hip: evolving indications. HSS J. 2013:9(1):60-9.

28. Mitsionis Gl, Lykissas MG, Motsis E, Mitsiou D, Gkiatas I, Xenakis TA, et al. Surgical management of posterior hip dislocations associated with posterior wall acetabular fracture: a study with a minimum follow-up of 15 years. J Orthop Trauma. 2012;26(8):460-5

\section{Publisher's Note}

Springer Nature remains neutral with regard to jurisdictional claims in published maps and institutional affiliations. 